\title{
New concept in assessing compactness of woven structure in terms of its resistivity
}

\author{
M. Tokarska ${ }^{1}$
}

Received: 9 January 2016/ Accepted: 15 March 2016/Published online: 21 March 2016

(c) The Author(s) 2016. This article is published with open access at Springerlink.com

\begin{abstract}
The main purpose of the study is to present a new concept in assessing impact of the woven fabric on its electroconductive properties. Fabric is treated as twocomponent composite i.e. anisotropic medium containing empty spaces filled with air shaped anisotropic ellipsoids. The method can be also used to assess the compactness, which is one of the features of Van der Pauw structure. The method allows one to determine resistivity of material composed of interlaced yarns as the material property. Analysis of chosen woven fabric structure was conducted. Percentage surface cover of the fabric by warp and weft yarns and depolarization factor were determined. It was found the woven fabrics satisfy requirements described in Van der Pauw structure. Using Van der Pauw method resistivity of material composed of interlaced yarns as a feature of the material was calculated for all woven fabrics. Resistivity of material is one of the required parameter in multiphysics simulation software used to understand physical phenomena of the designed object.
\end{abstract}

\section{Introduction}

Electroconductive textiles have wide possibilities of applications. They include heating elements $[1,2]$, electrical circuits [3, 4], antennas [5, 6], transmission lines [7, 8], electrodes [9, 10], electromagnetic shielding materials $[11,12]$. This kind of textiles can be made using conductive fibres. Naturally conductive fibers or metallic fibers are

M. Tokarska

magdalena.tokarska@p.lodz.pl

1 Department of Architecture of Textiles, Lodz University of Technology, 116 Zeromskiego St., 90-924 Lodz, Poland developed from electrically conductive metals by coating fibers with metals, conductive polymers, galvanic substances [13-15]. Electroconductive fibers can be incorporate with traditional non-conductive fibers to create electroconductive yarns. Conductive flat textile product can be made by coating techniques. Coatings can be applied to the surface of fibers, yarns or fabrics through processes including electroless plating, evaporative deposition, sputtering, and coating with a conductive polymer $[16,17]$. Electrical properties of flat textiles are influenced by properties of linear textile products, interaction between them (contact resistance) and their geometrical configuration affected geometrical shapes of inclusions.

Woven fabric can be compared to composite consisting of two constituents: conductive and dielectric materials. The magnitude of the metal permittivity is much larger than the permittivity of the dielectric component of the metal-dielectric composite [18]. Both, conductive materials and dielectrics are fibres and yarns. Distribution of the linear products creates system of empty spaces-inclusions filled with air. The voids are dielectrics and have a size relatively small compared to the characteristic dimensions of the porous material. They have also different shapes (open, closed, complex, targeted) and different dimensions determined by the Union of Pure and Applied Chemistry. IUPAC defines micropores as voids with a diameter of less than $2 \mathrm{~nm}$, mesopores having a diameter in the range from 2 to $50 \mathrm{~nm}$ and macropores with a diameter of more than $50 \mathrm{~nm}$. In macroscale, woven fabric voids belong to a group of macropores [19]. Whole macroscopic structure specimen consists of spatially repeated unit cell called woven fabric repeat. Anisotropic inclusions are insufficiently randomized in directions or forcibly oriented so that the preferred direction makes the composite is anisotropic [20]. The resistivity of the composite can lie anywhere 
between the resistivity of the components depending both on the volume fraction of components and the morphology of the medium [21].

There are four main classics of conductivity models. They include statistical thermodynamic, geometrical, and structure-oriented models described in detail by Lux [22]. Percolation and Bruggeman's effective media theories are used to electrical conductivity of components and combine most aspects of both percolation and effective media theories [23]. Percolation theory gives a phenomenological equation for the conductivity of composite medium near to a metal-insulator or conductor-perfect conductor transition. General effective media equation quantitatively fits electrical resistivity (conductivity) as a function the components volume fraction for two-component composites. Other inclusions properties, such as size and shape, can also have an effect on electroconductive properties of composite [24]. The potential range of considered inclusion shapes extends from flat disks to spheres to long needles [25].

McLachlan [26] proposed two equations to describe the resistivity of isotropic two-composite macroscopic composite as a function of the resistivity of the components, the volume fraction of each, the space dimension and a single morphology parameter, that determine a critical volume fraction [27]. The equations are interpolations between Bruggeman's symmetric and asymmetric media theories. The symmetric theory is based on a random mixture of spheres. The asymmetric theory is based on a dispersion of spheres of the one component coated with the other, host component. The more general case for oriented ellipsoids, with a depolarization factor (demagnetization coefficient) in the direction of current flow was discussed by Sen et al. [28]. The polarizability is a function of inclusion geometry, independent of volume, and orientation with respect to the applied electrical field [25]. Depolarization factor indicates the influence of the geometrical shape on the polarization of the dielectric ellipsoid in an electric field and the magnetization of the magnetic ellipsoid in a magnetic field [29]. A sphere has three the same depolarization factors (in directions $x, y, z)$ equal to $1 / 3$, a disc has depolarization factors equal to $1,0,0$ respectively, a needle has depolarization factors equal to $0,1 / 2,1 / 2$ respectively [30]. For a general ellipsoid with three different axes, the depolarization factors have to be calculated from the integral [30,31]. The internal static field of the ellipsoid, exposed to uniform electric field, is uniform even if the media are anisotropic [32].

Study of composite resistivity to understanding how different components material properties can change the resistivity values is important from the point of view of development of new composites for use in various applications.

In the article a new approach in assessing impact of the woven fabric structure on its electroconductive properties is presented. A new concept in assessing compactness of woven structure in terms of its resistivity is presented. Compactness is one of a feature of Van der Pauw structure [33]. The analysis can be used at the woven fabric design stage. It is possible to determine resistivity of material composed of interlaced yarns as a material property.

\section{Materials}

The electroconductive woven fabric specimens were chosen. Specimens S1 and S2 are made up of yarns manufactured from polyamide fibres metalized with silver. Specimens S3, S5, S6 and S7 are made up of yarns manufactured from polyester fibres metalized with nickel. Specimen S4 is made up of yarns manufactured from polyamide fibres metalized with silver, copper and tin. Structure parameters of the fabrics are summarized in Table 1. Measurements were repeated three times and the average value of each parameter was calculated.

Parameters of the fabrics were determined based on the following standards: ISO 5084 [34] to determine thickness and ISO 3801 [35] to determine mass per unit area. Number of warp and weft yarns was determined using Olympus SZX10 Stereo Microscope and Stream Motion software.

Additionally, morphometric measurements of fabrics images were conducted. Measurements were performed in three areas of each specimen, and then the average value of determined parameter was calculated. The following parameters of fabric structure were designated: the linear dimension of warp repeat $L_{w a}$, the linear dimension of weft repeat $L_{w e}$, the width of the warp yarns $d_{w a}$, the width of the weft yarns $d_{w e}$, the warp repeat $R_{w a}$ expressed in number of warp yarns in warp repeat and the weft repeat $R_{w e}$ expressed in number of weft yarns in weft repeat. The spacing of the warp yarns, $A_{w a}$, can be calculated as:

$A_{w a}=\frac{L_{w a}}{R_{w a}}$

The spacing of the weft yarns, $A_{w e}$, can be calculated as:

$A_{w e}=\frac{L_{w e}}{R_{w e}}$

Parameters are shown in Fig. 1.

Received results are summarized in Table 2. The spacing of the warp and weft yarns was calculated in according to formula (1) and (2), respectively.

\section{Methods}

The electrical conductivity of a composite is generally characterized by its dependence on inclusions volume fraction. The equivalents of dielectric inclusions in woven 
Table 1 Structure parameters of woven fabrics

\begin{tabular}{|c|c|c|c|c|c|}
\hline Specimen & Weave & $\begin{array}{l}\text { Thickness } \\
(\mathrm{mm})\end{array}$ & $\begin{array}{l}\text { Aerial density } \\
\left(\mathrm{g} / \mathrm{m}^{2}\right)\end{array}$ & $\begin{array}{l}\text { Warp yarns density } \\
(\text { number of yarns per } 1 \mathrm{~cm})\end{array}$ & $\begin{array}{l}\text { Weft yarns density } \\
\text { (number of yarns per } 1 \mathrm{~cm} \text { ) }\end{array}$ \\
\hline $\mathrm{S} 1$ & Plain & 0.148 & 76 & 41 & 31 \\
\hline $\mathrm{S} 2$ & Twill & 0.350 & 149 & 71 & 33 \\
\hline S3 & Twill & 0.294 & 155 & 45 & 34 \\
\hline $\mathrm{S} 4$ & Plain & 0.116 & 77 & 55 & 47 \\
\hline S5 & Plain & 0.078 & 75 & 40 & 32 \\
\hline S6 & Twill & 0.270 & 152 & 76 & 32 \\
\hline S7 & Plain & 0.154 & 113 & 29 & 25 \\
\hline
\end{tabular}

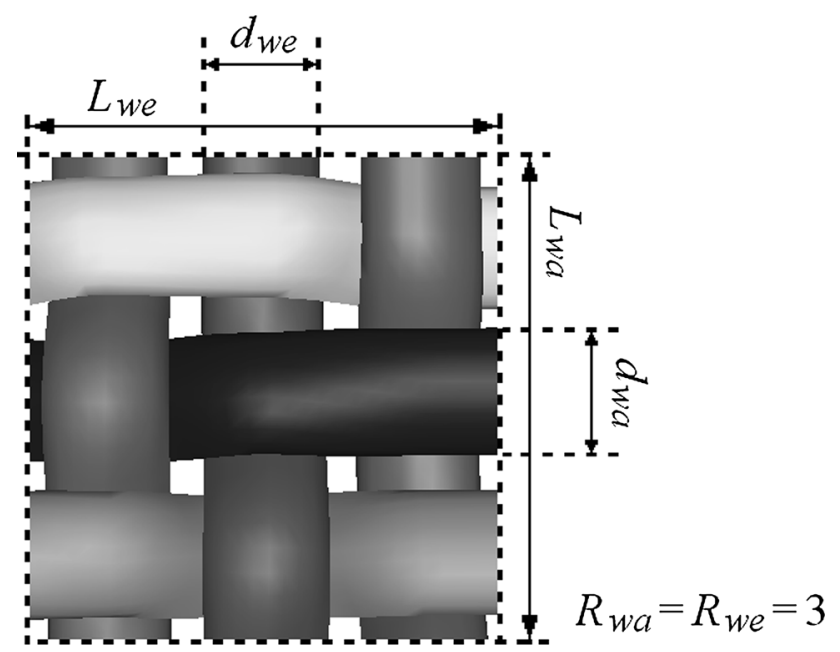

Fig. 1 Selected parameters for sample of fabric structure

fabric are voids evenly distributed within the specimen volume and subjected to a constant external electric field. Based on equation developed by McLachlan [27] resistivity $\rho_{c}$ of two-component composite type metal-dielectric is determined by the following formula:

$\rho_{c}=\rho_{m}\left(1-f_{p}\right)^{\frac{-1}{1-L}}$

where $\rho_{m}$ the resistivity of metal, $f_{p}$ the volume fraction of dielectric inclusions in the composite, $L$ the depolarization factor.
Formula (3) allows one to determine the conductivity of a medium consisting of two constituents, which together completely fill the media. The depolarization factor depends on the shape and geometry of the inclusions in the composite. Inclusions contained in anisotropic medium shaped anisotropic ellipsoids are assumed.

Let the ellipsoid has semi axes $a_{1}, a_{2}, a_{3}$ in directions $x$, $y, z$, respectively, and let the anisotropy of the medium be such that the permittivity components are $\varepsilon_{x}, \varepsilon_{y}, \varepsilon_{z}$ respectively. Then depolarization factors for directions of three principal axes of the ellipsoid are given by [31]:

$L_{i}=\frac{a_{1} a_{2} a_{3}}{2} \int_{0}^{\infty} \frac{d s}{\left(s+a_{i}^{2}\right) \sqrt{\left(s+a_{1}^{2}\right)\left(s+a_{2}^{2}\right)\left(s+a_{3}^{2}\right)}}$

wherein $\sum_{i=1}^{3} L_{i}=1$.

In order to calculate depolarization factors, $L_{1}, L_{2}, L_{3}$, for woven fabric, three semi axes $a_{1}, a_{2}, a_{3}$ of ellipsoid in directions $x, y, z$, respectively, can be calculated based on following dependences:

$a_{1}=0.5\left(A_{w a}-d_{w a}\right)$

$a_{2}=0.5\left(A_{w e}-d_{w e}\right)$

$a_{3}=0.5 h$

where $A_{w a}$ the spacing of the warp yarns, $A_{w e}$ the spacing of the weft yarns, $d_{w a}$ the width of the warp yarns, $d_{w e}$ the width of the weft yarns, $h$ the thickness of the woven fabric.
Table 2 Results of morphometric measurements of fabrics

\begin{tabular}{lllllllll}
\hline Specimen & $\begin{array}{l}d_{w a} \\
(\mathrm{~mm})\end{array}$ & $\begin{array}{l}L_{w a} \\
(\mathrm{~mm})\end{array}$ & $\begin{array}{l}R_{w a} \\
(\text { number of yarns })\end{array}$ & $\begin{array}{l}A_{w a} \\
(\mathrm{~mm})\end{array}$ & $\begin{array}{l}d_{w e} \\
(\mathrm{~mm})\end{array}$ & $\begin{array}{l}L_{w e} \\
(\mathrm{~mm})\end{array}$ & $\begin{array}{l}R_{w e} \\
(\text { number of yarns })\end{array}$ & $\begin{array}{l}A_{w e} \\
(\mathrm{~mm})\end{array}$ \\
\hline S1 & 0.21 & 0.48 & 2 & 0.24 & 0.28 & 0.64 & 2 & 0.32 \\
S2 & 0.14 & 0.84 & 6 & 0.14 & 0.27 & 1.80 & 6 & 0.30 \\
S3 & 0.18 & 0.66 & 3 & 0.22 & 0.27 & 0.87 & 3 & 0.29 \\
S4 & 0.16 & 0.36 & 2 & 0.18 & 0.20 & 0.42 & 2 & 0.21 \\
S5 & 0.23 & 0.50 & 2 & 0.25 & 0.27 & 0.62 & 2 & 0.31 \\
S6 & 0.13 & 0.65 & 5 & 0.13 & 0.28 & 1.55 & 5 & 0.31 \\
S7 & 0.25 & 0.68 & 2 & 0.34 & 0.33 & 0.80 & 2 & 0.40 \\
\hline
\end{tabular}


As a measure of the structure compactness of woven fabric, the percentage surface cover of the fabric by warp and weft yarns was adopted [36]. The percentage surface cover is given by:

$C_{t}=\frac{A_{w e} d_{w a}+A_{w a} d_{w e}-d_{w a} d_{w e}}{A_{w a} A_{w e}} \times 100$

Based on (8), the percentage surface fraction $f_{p}$ is defined as follows:

$f_{p}=1-0.01 C_{t}$

Thus, resistivity of woven fabric treated as two-component composite is determined by formula:

$\rho_{c}=\left(\frac{100}{C_{t}}\right)^{\frac{1}{1-L}} \rho_{m}$

where $\rho_{m}$ the resistivity of the woven fabric without inclusions, $L$ the depolarization factor, $C_{t}$ the percentage surface cover of the fabric by warp and weft yarns.

Resistivity $\rho$ of flat anisotropic specimen with its plane perpendicular to the direction of the principal resistivity $\rho_{3}$ in directions $x$ is equal to [37]:

$\rho=\sqrt{\rho_{c 1} \rho_{c 2} \rho_{c 3}}$

where $\rho_{c 1}, \rho_{c 2}, \rho_{c 3}$ the resistivities in directions $x, y, z$, respectively. Wasscher [37] proved that in the case of rectangular and circular flat specimens $\rho_{c 3}$ does not enter into the final results, thus formula (11) can be rewritten as:

$\rho=\sqrt{\rho_{c 1} \rho_{c 2}}$

Substituting formula (10) into (12) one finds that resistivity of the composite is:

$\rho_{c}=\rho_{m} \sqrt{m_{1} m_{2}}$

where

$m_{i}=\left(\frac{100}{C_{t}}\right)^{\frac{1}{1-L_{i}}}, \quad i=1,2$

On the other side, based on Wasscher's extension [37] of Van der Pauw method [33] to the case of anisotropic media, resistivity of circular specimen can be determined from equation:

$\rho_{c}=\pi h \cdot \sqrt{\frac{R_{1} R_{2}}{\ln \frac{2}{1-k} \ln \frac{2}{1+k}}}$

where $h$ the specimen thickness, $k$ the modulus of the complete elliptic integral of the first kind, $R_{1}$ the first resistance corresponding to one of the two directions of the main axes in the specimen plane, $R_{2}$ the second resistance corresponding to one of the two directions of the main axes in the specimen plane; wherein $R_{1}>R_{2}$ and the following formula is given:
$\frac{R_{1}}{R_{2}}=\frac{\ln \left(\frac{1-k}{2}\right)}{\ln \left(\frac{1+k}{2}\right)}$

Using formulae (13)-(16) the resistivity $\rho_{m}$ of the woven fabric without inclusions can be determined. Wherein specimen should have features described Van der Pauw structure [33].

\section{Analysis of woven fabric structure}

Structure of woven fabric and its shape of inclusions are described by factor $m$ described by formula (14). Dependences of $m$ on $L$ and $m$ on $C_{t}$ are shown in Fig. 2.

If the fabric is characterized with $100 \%$ surface cover, the $C_{t}$ is equal to 100 . Then $m=1$ the resistivity $\rho_{c}$ of the composite is the same as the resistivity $\rho_{m}$ of woven fabric (i.e. material without voids composed of interlaced yarns). If $C_{t}<100$, then the inclusions in woven fabric, may have significant impact on electroconductive properties of woven structure. Composite resistivity increases with decreasing percentage surface cover $C_{t}$ (Fig. 2a). Moreover, shape and dimensions of voids measured by depolarization factor $L$ also affect resistivity $\rho_{c}$ of twocomponent composite. Composite resistivity increases with increasing depolarization factor $L$ (Fig. 2b).

The structure compactness $C_{t}$ of chosen woven fabrics was calculated and summarized in Table 3. Values of percentage surface cover of the fabrics by warp and weft yarns are in the range of $[95.4,100.0]$.

Lengths of semi axes $a_{1}, a_{2}, a_{3}$ of ellipsoid, for woven fabrics, were calculated in according to formulae (5), (6) and (7). Measurement results summarized in Table 1 and Table 2 were used. Depolarization factors, $L_{1}, L_{2}, L_{3}$, for woven fabric were determined based on dependence (4). Solving Eq. (4) the value of depolarization factor in form of complex number was received. The real part was taken into account and given in Table 3.

The imaginary part, being of the order of magnitude $10^{-16}$ or less, was treated as numerical error and it was neglected. An example of $L_{1}$ calculation for specimen S1 using formula (4) and Mathematica ${ }^{\circledR}$ is shown in Fig. 3. The received numerical error is equal to $1.312 \times 10^{-16}$.

In case of fabrics S2 and S6 percentage surface cover $C_{t}$ equal to 100.0. It was found that the specimens haven't empty spaces filled with air. Therefore, depolarization factors, $L_{1}, L_{2}, L_{3}$, for the two woven fabrics weren't determined. Values of depolarization factors given in Table 3 are in the following ranges: $L_{1} \in[0.321,0.601], L_{2}$ $\in[0.283,0.657], L_{3} \in[0.018,0.178]$.

Next, values of three values of factor $m$, i.e.: $m_{1}$ $=m\left(L_{1}, C_{t}\right), m_{2}=m\left(L_{2}, C_{t}\right), m_{3}=m\left(L_{3}, C_{t}\right)$, were calculated for chosen fabrics. Received results are summarized 

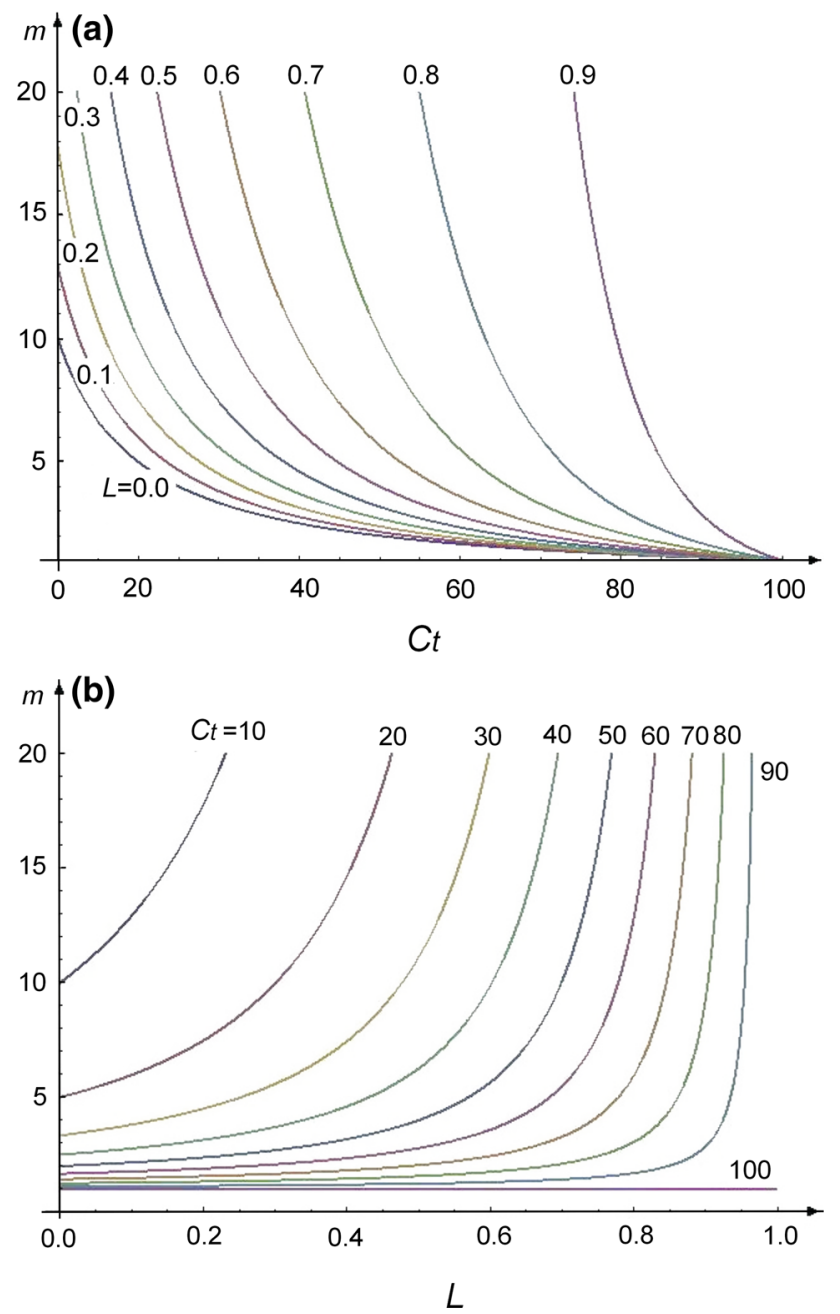

Fig. 2 a Dependence $m\left(C_{t}\right)$ for chosen values of $L=0.0,0.1,0.2$, $0.3,0.4,0.5,0.6,0.7,0.8,0.9$, $\mathbf{b}$ dependence $m(L)$ for chosen values of $C_{t}=10,20,30,40,50,60,70,80,90,100$

in Table 3. The effect of $C_{t}$ and $L$ on values of $m$ for specimens is shown in Fig. 4. Curves $m(L)$ shown in Fig. 4 are a part of a curves presented in Fig. $2 b$.

It was noted that values of factor $m$ doesn't exceed 1.1, which is caused in large part high fabric covering more than $95 \%$ (Table 3). In the case of fabrics S2 and S6 $C_{t}$ equals to 100.0 was obtained, therefore $m=1$. Depolarization factor depends on the shape and geometry of the specimen voids. The range of changes of depolarization factor values has less impact on $m$ factor than fabric surface cover.

\section{Determination of resistivity of woven fabrics}

Using Van der Pauw method the specimen should be thin and should have homogeneous and compactness structure [33]. Measures described the features were discussed in detail $[38,39]$. Based on the analysis measures of the characteristics typical for Van der Pauw structure were determined. Received results are juxtaposed in Table 4.

It was found the woven fabrics satisfy requirements described Van der Pauw structure. Measurements of resistances $R_{1}$ and $R_{2}$ were conducted in according to Van der Pauw method. Additionally quotient $R_{1} / R_{2}$ and ratio $R_{1} R_{2}$ were calculated for all the fabrics for the purposes of following calculations. Received results are presented in Table 5 .

In order to determine resistivity $\rho_{c}(15)$ of specimens the modulus $k$ was calculated using Mathematica ${ }^{\circledR}$. An example of $k$ calculation for specimen S1 using formula (16) and Mathematica ${ }^{\circledR}$ is shown in Fig. 5.

In according to formula (15) resistivity $\rho_{c}$ was calculated. Next, resistivity $\rho_{m}$ of material composed of interlaced yarns as the material property was determined using formula (13). Received results are presented in Table 6.

Received resistivity $\rho_{c}$ of materials is in the range of $\left[1.6 \times 10^{-6}, 6.7 \times 10^{-5}\right] \Omega \mathrm{m}$. Resistivity $\rho_{m}$ and $\rho_{c}$ are comparable. This is due to the fact that the value of the square root occurs in formula (13) is close to unity. This is connected with high percentage surface cover of the woven fabrics by warp and weft yarns. Received resistivity $\rho_{m}$ of materials is in the range of $\left[1.6 \times 10^{-6}, 6.5 \times 10^{-5}\right] \Omega \mathrm{m}$. So the interlaced yarns have electrical resistivity similar to metals. Resistivity of material is one of the required parameter in multiphysics simulation software used to understand physical phenomena of the designed object.
Table 3 Factors obtained for woven fabrics with voids shaped ellipsoids

\begin{tabular}{lrlllllllll}
\hline Specimen & \multicolumn{1}{c}{$C_{t}$} & $\begin{array}{l}a_{1} \\
(\mathrm{~mm})\end{array}$ & $\begin{array}{l}a_{2} \\
(\mathrm{~mm})\end{array}$ & $\begin{array}{l}a_{3} \\
(\mathrm{~mm})\end{array}$ & $\begin{array}{l}L_{1} \\
-\end{array}$ & $\begin{array}{l}L_{2} \\
-\end{array}$ & $\begin{array}{l}L_{3} \\
-\end{array}$ & $\begin{array}{l}m_{1} \\
-\end{array}$ & $\begin{array}{l}m_{2} \\
-\end{array}$ & $\begin{array}{l}m_{3} \\
-\end{array}$ \\
\hline S1 & 98.4 & 0.015 & 0.020 & 0.074 & 0.535 & 0.396 & 0.069 & 1.035 & 1.027 & 1.017 \\
S2 & 100.0 & 0.000 & 0.015 & 0.175 & - & - & - & 1.000 & 1.000 & 1.000 \\
S3 & 98.7 & 0.020 & 0.010 & 0.147 & 0.325 & 0.657 & 0.018 & 1.020 & 1.039 & 1.013 \\
S4 & 99.5 & 0.010 & 0.005 & 0.058 & 0.321 & 0.652 & 0.027 & 1.007 & 1.015 & 1.005 \\
S5 & 99.0 & 0.010 & 0.020 & 0.039 & 0.601 & 0.283 & 0.116 & 1.026 & 1.014 & 1.011 \\
S6 & 100.0 & 0.000 & 0.015 & 0.135 & - & - & - & 1.000 & 1.000 & 1.000 \\
S7 & 95.4 & 0.045 & 0.035 & 0.077 & 0.354 & 0.468 & 0.178 & 1.076 & 1.093 & 1.059 \\
\hline
\end{tabular}


$\ln [2]:=$ DepFactor $\left[a I_{-}, a 2_{-}, a 3_{-}\right]:=$ Module $\left[\{\right.$ calculation $\}$, calculation $\left.=\frac{a 1 * a 2 * a 3}{2} \int_{0}^{\infty} \frac{1}{\left(s+a I^{2}\right) \sqrt{\left(s+a I^{2}\right)\left(s+a 2^{2}\right)\left(s+a 3^{2}\right)}} d s\right]$

DepFactor $[0.015,0.020,0.074]$

Out $[3]=0.535449+1.3124 \times 10^{-16}$ i

Fig. 3 An example of $L_{1}$ calculation for specimen $S 1$ in Mathematica ${ }^{\circledR}$

Fig. 4 Effect of woven fabric surface cover and depolarization factor on $m$ factor

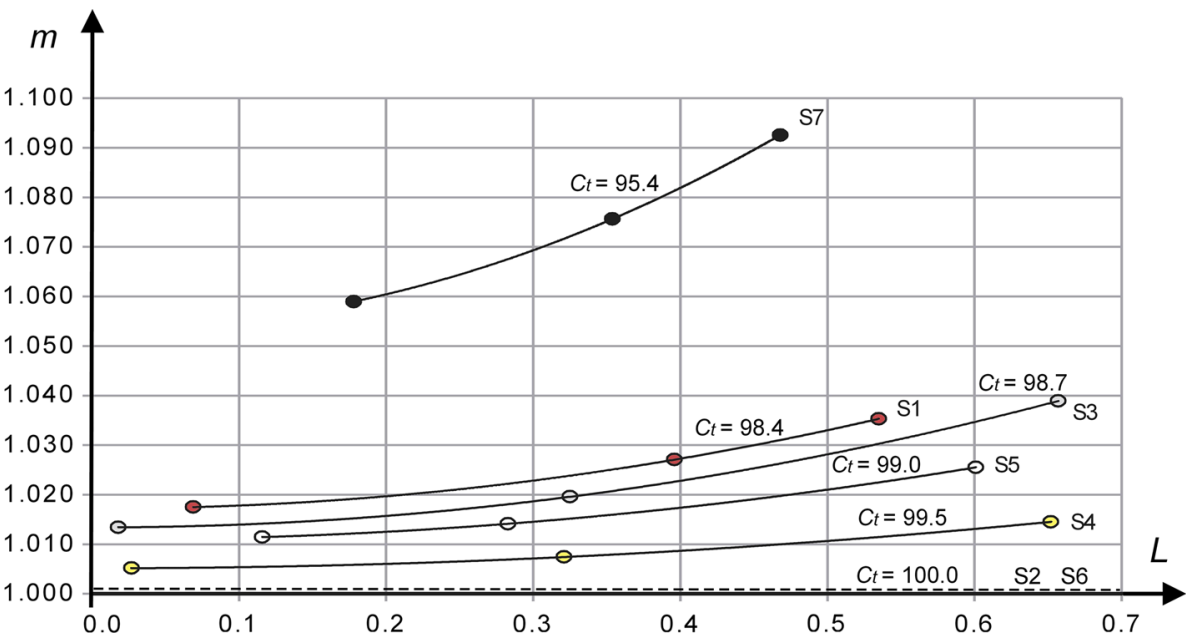

Table 4 Measures of the characteristics typical for Van der Pauw structure

\begin{tabular}{|c|c|c|c|c|}
\hline Specimen & Condition $\rightarrow$ & $\begin{array}{l}\text { Specimen thickness }{ }^{\mathrm{a}} h(\mathrm{~mm}) \\
h \leq 1.000 \mathrm{~mm}\end{array}$ & $\begin{array}{l}\text { Structure compactness }{ }^{\mathrm{b}} C_{t}(-) \\
C_{t} \geq 95.0\end{array}$ & $\begin{array}{l}\text { Structure homogeneity }{ }^{\mathrm{c}} H_{t}(-) \\
H_{t} \leq 0.001\end{array}$ \\
\hline $\mathrm{S} 1$ & & 0.148 & 98.4 & 0.00004 \\
\hline $\mathrm{S} 2$ & & 0.350 & 100.0 & 0.00019 \\
\hline S3 & & 0.294 & 98.7 & 0.00007 \\
\hline $\mathrm{S} 4$ & & 0.116 & 99.5 & 0.00002 \\
\hline S5 & & 0.078 & 99.0 & 0.00004 \\
\hline S6 & & 0.270 & 100.0 & 0.00013 \\
\hline S7 & & 0.154 & 95.4 & 0.00007 \\
\hline
\end{tabular}

${ }^{\text {a }}$ See Table 1

b See Table 3

c Measure of structure homogeneity is calculated based on equation $H_{t}=A_{w a} R_{w a} A_{w e} R_{w e} / S$, where $S=7854 \mathrm{~mm}^{2}$ - surface of tested circular specimen of $100 \mathrm{~mm}$ in diameter [38]

Table 5 Measurements results

\begin{tabular}{lllll}
\hline Specimen & $\begin{array}{l}R_{1} \\
(\Omega)\end{array}$ & $\begin{array}{l}R_{2} \\
(\Omega)\end{array}$ & $\begin{array}{l}R_{1} / R_{2} \\
(-)\end{array}$ & $\begin{array}{l}R_{1} R_{2} \\
\left(\Omega^{2}\right)\end{array}$ \\
\hline S1 & 0.037 & 0.023 & 1.61 & 0.00085 \\
S2 & 0.020 & 0.014 & 1.43 & 0.00028 \\
S3 & 0.069 & 0.035 & 1.97 & 0.00242 \\
S4 & 0.004 & 0.002 & 2.00 & 0.00001 \\
S5 & 0.006 & 0.003 & 2.00 & 0.00002 \\
S6 & 0.052 & 0.036 & 1.44 & 0.00187 \\
S7 & 0.007 & 0.006 & 1.17 & 0.00004 \\
\hline
\end{tabular}

\section{Conclusions}

A new concept in assessing compactness of woven structure in terms of its resistivity can be used to assess the compactness, one of a feature of Van der Pauw structure.

Percentage surface cover of fabrics by warp and weft yarns was calculated. Values in the range of $[95.4,100.0]$ were received. Moreover it was found that the composite resistivity increases with decreasing percentage surface cover. 


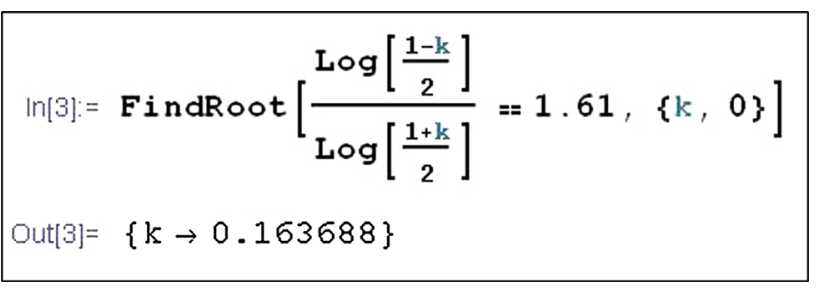

Fig. 5 An example of $k$ calculation for specimen $\mathrm{S} 1$ in Mathematica ${ }^{\circledR}$

Table 6 Results of calculation of fabrics resistivity

\begin{tabular}{lllll}
\hline Specimen & $\begin{array}{l}k \\
(-)\end{array}$ & $\begin{array}{l}\rho_{c} \\
(\Omega \mathrm{m})\end{array}$ & $\begin{array}{l}\left(m_{1} m_{2}\right)^{0.5} \\
(-)\end{array}$ & $\begin{array}{l}\rho_{m} \\
(\Omega \mathrm{m})\end{array}$ \\
\hline S1 & 0.164 & $2.0 \times 10^{-5}$ & 1.03 & $1.9 \times 10^{-5}$ \\
S2 & 0.123 & $2.7 \times 10^{-5}$ & 1.00 & $2.7 \times 10^{-5}$ \\
S3 & 0.231 & $6.7 \times 10^{-5}$ & 1.03 & $6.5 \times 10^{-5}$ \\
S4 & 0.236 & $1.7 \times 10^{-6}$ & 1.01 & $1.7 \times 10^{-6}$ \\
S5 & 0.236 & $1.6 \times 10^{-6}$ & 1.02 & $1.6 \times 10^{-6}$ \\
S6 & 0.126 & $5.3 \times 10^{-5}$ & 1.00 & $5.3 \times 10^{-5}$ \\
S7 & 0.054 & $4.4 \times 10^{-6}$ & 1.08 & $4.1 \times 10^{-6}$ \\
\hline
\end{tabular}

Depolarization factor of fabrics was determined. Values in the ranges of [0.321, 0.601], [0.283, 0.657], [0.018, $0.178]$ in directions $x, y, z$ were obtained respectively. It was found that the composite resistivity increases with increasing depolarization factor.

It was found the chosen woven fabrics satisfy requirements described Van der Pauw structure.

Using Van der Pauw method resistivity of anisotropic woven fabrics was calculated. Values in the range of $\left[1.6 \times 10^{-6}, 6.7 \times 10^{-5}\right] \Omega \mathrm{m}$ were received.

Resistivity of textile material composed of interlaced yarns as the material property was calculated for all woven fabrics. Received resistivity $\rho_{m}$ of materials is in the range of $\left[1.6 \times 10^{-6}, 6.5 \times 10^{-5}\right] \Omega \mathrm{m}$. So the interlaced yarns have electrical resistivity similar to metals.

\section{Complaince with ethical standards}

Conflict of interest The authors declare that they have no conflict of interest.

Open Access This article is distributed under the terms of the Creative Commons Attribution 4.0 International License (http://creati vecommons.org/licenses/by/4.0/), which permits unrestricted use, distribution, and reproduction in any medium, provided you give appropriate credit to the original author(s) and the source, provide a link to the Creative Commons license, and indicate if changes were made.

\section{References}

1. A. Kurczewska, J. Leśnikowski, Int. J. Occup. Saf. Ergon. 14, 77 (2008)
2. H. Özdemir, S. Kılınç, Autex Res. J. 15, 99 (2015)

3. I. Locher, G. Tröster, Text. Res. J. 78, 583 (2008)

4. B. Karaguzel, C.R. Merritt, T. Kang, J.M. Wilson, H.T. Nagle, E. Grant, B. Pourdeyhimi, J. Text. Inst. 100, 1 (2009)

5. R. Salvado, C. Loss, R. Gonçalves, P. Pinho, Sensors 12, 15841 (2012)

6. I. Kazani, F. Declercq, M.L. Scarpello, C. Hertleer, H. Rogier, D. Vande Ginste, G. De Mey, G. Guxho, L. Van Langenhove, Autex Res. J. 14, 47 (2014)

7. D. Cottet, J. Grzyb, T. Kirstein, G. Tröster, IEEE Trans. Adv. Packag. 26, 182 (2003)

8. J. Leśnikowski, M. Tokarska, Text. Res. J. 84, 290 (2014)

9. H.-Y. Song, J.-H. Lee, D. Kang, H. Cho, H.-S. Chuo, J.-W. Lee, Y.-J. Lee, J. Text. Inst. 101, 758 (2010)

10. J. Zięba, M. Frydrysiak, M. Tokarska, Fibres Text. East. Eur. 19, 70 (2011)

11. S. Tezel, Y. Kavuşturan, G.A.E. Vandenbosch, V. Volski, Text. Res. J. 84, 461 (2014)

12. Z. Stempień, T. Rybicki, E. Rybicki, M. Kozanecki, M.I. Szynkowska, Synth. Met. 202, 49 (2015)

13. V. Šafářová, J. Militký, J. Mater. Sci. Eng. B 2, 197 (2012)

14. M. Mirjalili, L. Karimi, Autex Res. J. 15, 87 (2015)

15. K. Cherenack, L. van Pieterson, J. Appl. Phys. 112, 091301 (2012)

16. S. Jiang, D. Miao, G. Yang, Z. Chen, A. Li, S. Shang, J. Mater. Sci. Mater. Electron. 26, 3364 (2015)

17. R. Pawlak, E. Korzeniewska, M. Frydrysiak, J. Zięba, Ł. Tęsiorowski, K. Gniotek, Z. Stempień, M. Tokarska, Fibres Text. East. Eur. 20, 68 (2012)

18. W. Cai, V. Shalaev, Optical Metamaterials: Fundamentals and Applications (Springer, New York, 2010)

19. M. Kuhr, D. Aibibu, Ch. Cherif, J. Ind. Text. 45, 853 (2016)

20. L.A. Apresyan, D.V. Vlasov, Tech. Phys. 59, 1760 (2014)

21. N. Deprez, D.S. McLachlan, J. Phys. D Appl. Phys. 21, 101 (1988)

22. F. Lux, J. Mater. Sci. 28, 285 (1993)

23. D.S. McLachlan, M. Blaszkiewicz, R.E. Newnham, J. Am. Ceram. Soc. 73, 2187 (1990)

24. M.L. Clingerman, Development and Modelling of Electrically Conductive Composite Materials (Michigan Technological University, Houghton, 2001)

25. S.B. Jones, S.P. Friedman, Water Resour. Res. 36, 2821 (2000)

26. D.S. McLachlan, J. Phys. C Solid State Phys. 19, 1339 (1986)

27. D.S. McLachlan, J. Phys. C Solid State Phys. 20, 865 (1987)

28. P.N. Sen, C. Scala, M.H. Cohen, Geophysics 46, 781 (1981)

29. F. Duan, J. Guojun, Introduction to Condensed Matter Physics, vol. 1 (World Scientific, Singapore, 2005)

30. A. Sihvola, J. Nanomater. 2007, 1 (2007)

31. L.D. Landau, E.M. Lifshitz, Electrodynamics of Continuous Media. Course of Theoretical Physics, vol. 8 (Pergamon, New York, 1960)

32. A.H. Sihvola, I.V. Lindell, AEU Int. J. Electron. Commun. 50, 289 (1996)

33. L.J. Van der Pauw, Philips Res. Rep. 13, 1 (1958)

34. ISO 5084:1996. Textiles-Determination of thickness of textiles and textile products

35. ISO 3801:1977. Textiles-Woven fabrics-Determination of mass per unit length and mass per unit area

36. J. Szosland, Autex Res. J. 3, 102 (2003)

37. J.D. Wasscher, Electrical Transport Phenomena in MnTe, An Antiferromagnetic Semiconductor (Philips' Gloeilampenfabriken, Eindhoven, 1969)

38. M. Tokarska, Autex Res. J. 14, 55 (2014)

39. M. Tokarska, K. Gniotek, J. Text. Inst. 106, 9 (2015) 